\title{
DOES INTRAVENOUS MIDAZOLAM INDUCE HYPERALGESIA?
}

\section{Coimbra L ${ }^{\mathbf{1}}$, Fernandes $\mathbf{A}^{\mathbf{1}}$, Gouveia $\mathrm{F}^{\mathbf{1}}$, Alves $\mathbf{L}^{\mathbf{2}}$, Amaro $\mathbf{L}^{\mathbf{1}}$, Dahlem $\mathbf{C}^{\mathbf{1}}$}

${ }_{1}^{1}$ Department of Anaesthesiology, Centro Hospitalar Vila Nova de Gaia/Espinho, EPE 2 USF Santo André de Canidelo, Vila Nova de Gaia; Saúde Comunitária, Escola de Ciências da Saúde, Universidade do Minho, Portugal

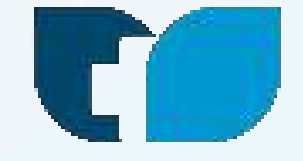

CENTRO

HOSPITALAR

\section{BACKGROUND \& AIMS}

Benzodiazepines are often prescribed to surgical patients as an anxiolytic premedication, but its effect on nociception is still obscure. Studies in rats have suggested a hyperalgesic effect of high doses of systemically administered midazolam, probably through a mechanism involving other than GABA-A receptors, or through nociception modulation ${ }^{1}$, but this effect is still to be proven in humans. This study aimed at assessing how intravenous (IV) midazolam affected patient outcome of ambulatory arthroscopic knee surgery (AAKS).

\section{MATERIAL \& METHODS}

This observational, retrospective study included all patients submitted to AAKS under spinal (S) anaesthesia between Jan. 2011 and Dec. 2015. Demographic data, drugs administered and $24 \mathrm{~h}$ PO pain scores and functional status (by telephone interview) were recorded. Association between midazolam dose and pain and limitations was estimated by logistic regression models, adjusting for age and ASA status. Significance level was defined as $p<0,05$. Ethical approval has been granted.

\begin{tabular}{|c|c|c|c|c|c|}
\hline \multicolumn{6}{|c|}{ RESULTS } \\
\hline 314 patients & Variables & Patients ( $n=314$ ) & Included $(n=270)$ & Excluded ( $n=39)$ & $p$-value \\
\hline \multirow{6}{*}{$\begin{array}{l}\text { Excluded: } \\
\text { - } 39 \text { due to lack of data } \\
\text { - } 5 \text { due to combined } S \text { and femoral block }\end{array}$} & \multirow{2}{*}{ Gender } & Male (M) & $151(55,9 \%)$ & $23(59 \%)$ & \multirow{2}{*}{0,720} \\
\hline & & Female $(F)$ & $119(44,1 \%)$ & $16(41 \%)$ & \\
\hline & Age & Years, mean \pm SD & $52,2( \pm 9,76)$ & $50,1( \pm 11,91)$ & 0,2167 \\
\hline & \multirow{3}{*}{ ASA status } & 1 & $62(23 \%)$ & $8(20,5 \%)$ & \multirow{3}{*}{0,391} \\
\hline & & 2 & $197(73 \%)$ & $31(79,5 \%)$ & \\
\hline & & 3 & $11(4 \%)$ & 0 & \\
\hline
\end{tabular}

Anaesthesia: spinals performed in the lateral position with hyperbaric bupivacaíne (mean \pm SD: $9,2 \pm 1,26 \mathrm{mg}$ ). 9 patients also received intrathecal sufentanyl (1-2,5 ug), and other 9 IV fentanyl (50-150 ug) as a pre-medication. Total dose of IV midazolam varied between 0 and $8 \mathrm{mg}$.

\begin{tabular}{|c|c|c|c|c|c|c|}
\hline $\begin{array}{c}\text { Graphic } 1 \quad \text { Pain scores at 24h Po } \\
120 \\
100 \\
80 \\
60 \\
40 \\
20 \\
0\end{array}$ \\
\hline
\end{tabular}

Higher doses of midazolam increased the likelihood of having PO pain in men (table 2.), but not in women, and this effect was independent of other variables; higher doses of midazolam were also linked to more PO functional limitations, but without statistical significance.

\section{There was no statistical difference regarding} the use of opioid and PO pain or limitations.

There was no statistical difference between different doses of local anesthetic and PO pain or limitations.

Table 2. Doses of midazolam and presence of pain/functional limitations at $24 \mathrm{~h} \mathrm{PO}$

\section{DISCUSSION}

Trials in mice have suggested both analgesic and hyperalgesic effects of midazolam, and different results seem to be related to different sites of action at the spinal cord or brain. ${ }^{2,3}$ This hyperalgesic effect is not clear in humans.

Limitations of our study:

- Observational and retrospective study - some data might have been lost or incorrectly collected.

- Perioperative anxiety levels not evaluated - could influence the midazolam doses and how patients feel their pain.

- Few patients receiving opioids - might justify why there was no impact on PO pain.

- Pain not evaluated preoperatively neither at the end of surgery - previous pain contributes to $P O$ pain.

Possible bias: do men with less pain tolerance need more midazolam for anxiety control intraoperatively, and will complain more the following day?

\section{CONCLUSION}

Optimal pain control and quick functional recovery are desired outcomes in ambulatory surgery. Our results suggest a statistically significant association between higher midazolam doses and more PO pain, but this effect arised only in men and its causality is still to be clarified. Further studies are needed to establish if the hyperalgesic effect described in rats is also seen in humans.

\section{REFERENCES}

1. Koch SC. Fitzgerald M. Hathway GJ. . Midazolam potentiates nociceptive behavior, sensitizes cutaneous reflexes, and is devoid of sedative action in neonatal rats. Anesthesiology. 2008 Jan;108(1):122-9. 